\title{
«Das ist eine andere Medizin»
}

\section{Daniel Lüthi}

Freier Journalist und Fotograf, Medientrainer, Bern

Es ist eine andere Art von Arztpraxis: Überall hat es Schnappschlösser - wegen der Sicherheit. Gleichzeitig duzen sich Ärzte und Patienten bisweilen, was auf eine ungewöhnliche Vertrautheit schliessen lässt. Die Sprechstunden werden in mindestens drei Sprachen, oder auch mit Dolmetscher durchgeführt. Mehrheitlich sind es Leute von ganz unten, die hier ein- und ausgehen: suchtkranke Menschen oder Sexarbeiterinnen, Flüchtlinge und Asylbewerber. Cyrille Bocion schätzt die Vielseitigkeit, die Vielfarbigkeit hier. Aber ab und zu stösst auch er an Grenzen.

\section{Menschen in prekären Situationen}

«Wir betreuen nicht nur marginalisierte Menschen, sondern schon auch 'gewöhnliche' Patienten wie andere
Arztpraxen", sagt er. Gemeint sind Leute, die in der Nähe wohnen, oder solche, die früher im MethadonProgramm waren und geblieben sind. Auch für Touristen übernimmt das Ambulatorium die Rolle einer «normalen» Hausarztpraxis - für Leute also, die zum Vergnügen auf der Reise sind.

Die meisten Klientinnen und Klienten hier jedoch sind, wie die Stadt schreibt, «Menschen in prekären Lebenssituationen». Viele von ihnen kommen via Sozialamt: Obdachlose ohne Krankenkasse, Sexarbeiterinnen von der nahen Langstrasse.

Rund ein Drittel des Klientels ist suchtkrank. «Ein paar von ihnen sind verheiratet, haben Kinder. Sie haben es geschafft, sich ein stabiles Umfeld einzurichten", erzählt Bocion. Vor 25 oder 30 Jahren drohten sie vielleicht im «Needle Park» auf dem Platzspitz unterzugehen.

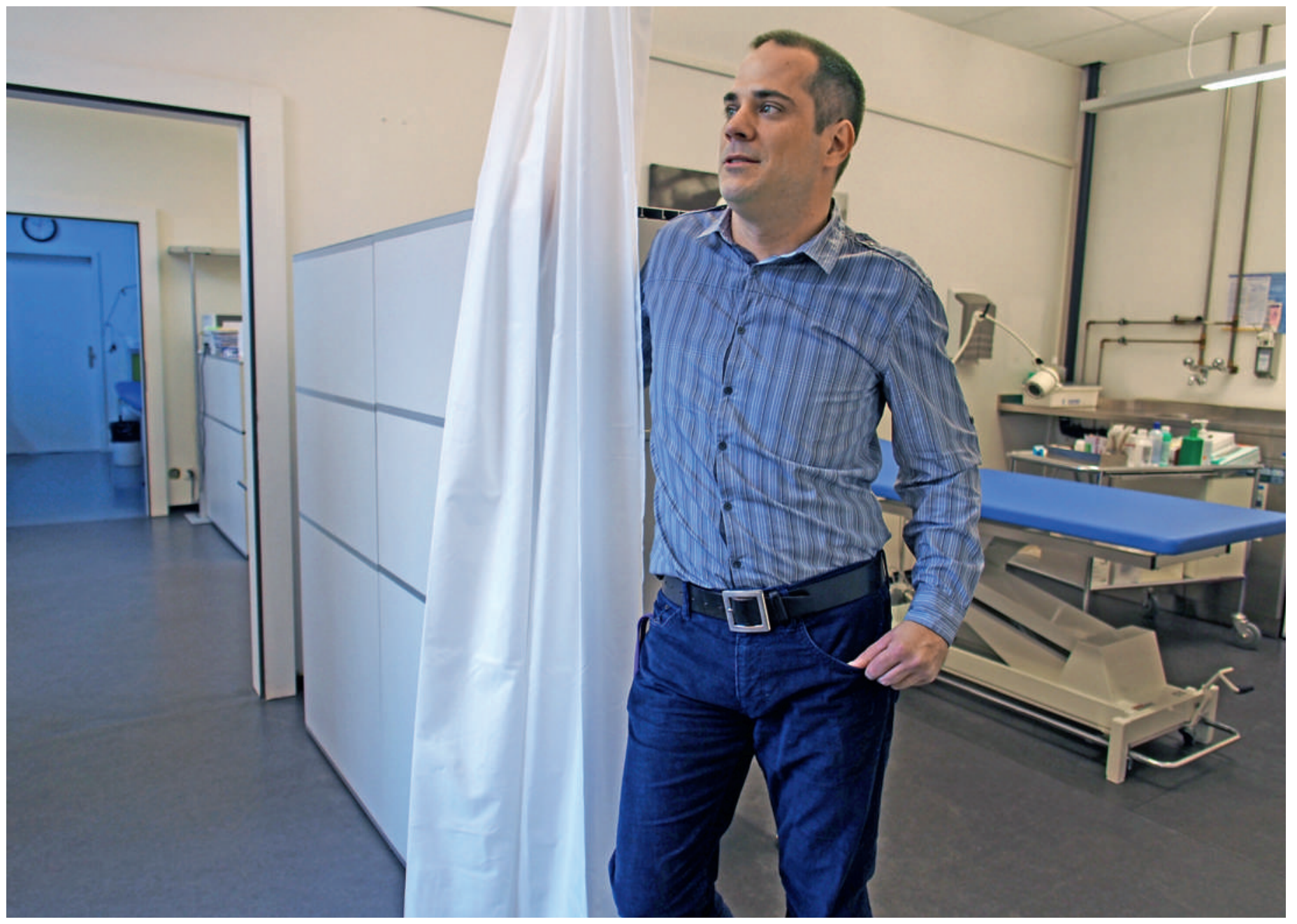


Als Cyrille Bocion 1995 nach Zürich kam, schloss die Polizei gerade die Drogenhölle am Oberen Letten. Er kennt das Elend von damals also nur aus Erzählungen - und behandelt heute dessen Spätfolgen.

"Es ist unfassbar, was diese Leute erzählen", sagt Bocion, "das muss wie im Krieg gewesen sein. Heute geht es diesen Leuten wesentlich besser. Durch die jahrelange Suchterkrankung kennen sie die Grenzen ihres Körpers meist genau.» So habe er denn auch keine grossen Bedenken, wenn sich einer nicht strikt an das halte, was er aus medizinischer Sicht empfehle. «Die wissen, was sie brauchen.»

Kürzlich habe er einen 50-jährigen Mann mit einer starken Lungenentzündung und einem Pneumothorax empfangen. «Er konnte fast nicht mehr atmen. Aber für ihn selber war seine Situation nicht schlimm genug für eine Hospitalisation. Er wollte unbedingt noch etwas konsumieren, und er wusste, dass dies im Spital nicht möglich sein wird.» Zweierlei illustriere das Beispiel, sagt Bocion: "Wie dominant eine Sucht sein kann, und wie viel diese Menschen schon erlebt haben, wie hoch deshalb also ihre Toleranzschwelle ist.»

\section{Menschen auf der Flucht}

Seit fast genau zwei Jahren kommen hierher zahlreiche Menschen aus aller Welt, die nicht vergnügungshalber unterwegs sind, sondern auf der Flucht vor Krieg, Hunger, Verfolgung.

Ganz in der Nähe des Ambulatoriums, in Zürich-Altstetten, wird seit Januar 2014 das beschleunigte Verfahren für Asylbewerber getestet. Für die rund $350 \mathrm{Be}$ wohnerinnen und Bewohner dieses sogenannten Testzentrums ist der stadtärztliche Dienst medizinischer Gatekeeper, das Ambulatorium also quasi die zuständige Hausarztpraxis.

\section{«Es ist unfassbar, was diese Leute erzählen.»}

Viele Flüchtlinge leiden bei ihrer Ankunft unter Parasiten, aktuell ist beispielsweise die Wurmkrankheit Bilharziose. «Relativ oft sehen wir Malaria-Patienten. Diese schicken wir zur weiteren Behandlung direkt ins Spital. Und immer wieder gibt es auch mögliche Tuberkulose-Fälle. Diese Personen werden von Spezialisten der Lungenliga abgeklärt.» Dazu kommen ein paar HIV- sowie Hepatitis-B- und -C-Patienten. Courant normal also für einen Arzt in einem solchen Ambulatorium. Und trotzdem: Was Cyrille Bocion mit dieser Klientengruppe erlebt, ist bisweilen ebenso unfassbar wie die Erzählungen aus den Zeiten der offenen Drogenszene beim Platzspitz oder Letten.

\section{Cyrille Bocion}

Med. Pract. Cyrille Bocion wurde 1975 in Nyon geboren. Dort besuchte er auch die Schulen. Mit 20 kam er nach Zürich, wo er zuerst Veterinär-, und dann Humanmedizin studierte. 2007 machte er sein Staatsexamen. Am Zürcher Waidspital bildete er sich in Geriatrie weiter, in Thusis arbeitete er in der Chirurgie, in Winterthur in der Inneren Medizin. Seit 2012 ist er im medizinischen Ambulatorium der Stadt Zürich tätig, im Areal der Alten Kaserne an der Kanonengasse. Seit Anfang dieses Jahres ist er dort stellvertretender Oberarzt. Cyrille Bocion ist ledig. Er wohnt in Zürich.

Da kam letzthin zum Beispiel eine Frau aus Eritrea mit starken Schmerzen im Schulterbereich. «Die Frau wurde auf ihrer Odyssee zusammengeschlagen und vergewaltigt, sie war dann drei Monate lang mit einem gebrochenen Schlüsselbein unterwegs», konnte Cyrille Bocion in Erfahrung bringen. "Aber auf ihrem langen Weg werden die Flüchtlinge meist kaum medizinisch versorgt. Wir sehen viele, die noch nie einen Arzt gesehen haben.»

\section{Grenzen}

Die Flüchtlinge stammen zum Beispiel aus Somalia und Syrien, aus Sri Lanka und der Ukraine. "An zwei Tagen pro Woche haben wir eine reguläre Sprechstunde - einige Zusätzliche kommen unangemeldet.» Und mit diesen Menschen, die viele Grenzen überquert haben, lernt Cyrille Bocion manchmal seine eigenen Grenzen kennen. Der erste Termin daure 30 Minuten, berichtet er. Jeder weitere Termin 20 Minuten. «In dieser kurzen Zeit kann ich häufig nur Symptome abklären, eine Blutentnahme, ein Röntgen machen. Es ist unmöglich, in eine Geschichte einzutauchen, und ich versuche dies auch zu vermeiden. Denn starke psychische Reaktionen könnte ich während der laufenden Sprechstunden nicht auffangen.» Falls es doch zu solchen Belastungsreaktionen komme, versuche er, sobald als möglich einen Termin in der psychiatrischen Sprechstunde zu vereinbaren, die das Ambulatorium ebenfalls anbietet. «Psychische Erkrankungen, insbesondere eine posttraumatische Belastungsstörung, stellen eine grosse Herausforderung dar», sagt Bocion. «Oft fehlen Therapieplätze, um die Betroffenen nach der Diagnostik zu behandeln.»

Dazu komme, dass diese Menschen ja bloss 140 Tage im Testzentrum bleiben können. Und: «Immer wieder tauchen Flüchtlinge unter oder kehren selbständig in ihr Heimatland zurück, bevor die Abklärungen abgeschlossen sind.» 
Mit anderen Worten: Oft kann dieser Arzt dem, was er gelernt hat und auch umsetzen möchte, nicht gerecht werden - ebenso wenig wie seinen Ansprüchen an sich selber. "Zusammen mit der zeitweise überdurchschnittlichen Arbeitsbelastung - zeitlich, inhaltlich und psychisch - kommt man manchmal an seine Grenzen", sagt Cyrille Bocion.

Aber er sagt auch: "Ich arbeite gerne hier.» Weil er etwas Ähnliches erlebe, wie in seiner Ausbildungszeit in der Geriatrie: «Auch dort spielte nicht nur das Medizinische eine Rolle, sondern auch das Soziale. Das ist eine andere Medizin. In einem Spital beispielsweise sieht man oft keinen Verlauf, da werden Patienten behandelt und wieder nach Hause geschickt. Später kommen sie dann unter Umständen mit dem gleichen Leiden wieder.»

\section{"Ich wünsche mir, dass menschliche und medizinische Kriterien wichtiger werden.»}

Hier, im Ambulatorium, spielen auch kulturelle und religiöse Unterschiede eine grosse Rolle. Einfühlungsvermögen und Fingerspitzengefühl sind besonders gefragt. Ein Beispiel: «Einige Patienten wollen kein Blut geben, weil sie gelernt haben, dass Blut Leben ist. Sie meinen also, sie würden ihr Leben geben, wenn wir ihnen Blut nehmen», berichtet Bocion.

\section{Festtage?}

Die Weihnachtszeit ist eine Zeit der Wünsche. Für sich selber wünscht sich Cyrille Bocion etwas mehr Zeit. Gerne würde er ab und zu schwimmen oder joggen gehen, würde er sich mehr mit Freunden treffen. «Solche Dinge kommen eindeutig zu kurz.» Für die Patientinnen und Patienten aus den verschiedenen Welten sind die Festtage in der Regel keine Festtage, sondern eine besonders schwierige Zeit. "Wir offerieren Kekse, geben Einkaufsgutscheine ab und beraten sie, wohin sie in diesen besonderen Tagen gehen können. Die Stiftung Pfarrer Sieber ist nach wie vor eine gute Adresse», sagt Bocion.

Auch ihnen, seinen Patientinnen und Patienten, wünscht er sich mehr Zeit. Besonders denen, die auf der Flucht sind: «Dass sie länger bleiben und wir besser abklären und behandeln können", sagt Bocion. Und zwar auch deshalb, "weil der Flüchtlingsstrom ja immer grösser wird und dies wohl auch die Schweiz zu spüren bekommt». Und wenn es die Schweiz zu spüren bekommt, spüren es sehr früh auch Cyrille Bocion und seine Kolleginnen und Kollegen im Ambulatorium an der Kanonengasse. Eine Adresse, die in diesem Zusammenhang eine spezielle Note erhält.

"Viele unserer Patientinnen und Patienten waren lange auf der Flucht. Deshalb haben sie lange auf einen Arztbesuch warten müssen. Wenn wir ihnen jetzt das Gefühl vermitteln, sie nicht ernst zu nehmen, ist das besonders schlimm. Sie können ja nicht einfach den Arzt wechseln.»

Cyrille Bocion hält inne. Er lässt seinen Blick ins neblige Kasernenareal schweifen und sagt dann: «Ich wünsche mir, dass die Leute da draussen ihre Augen mehr öffnen. Dass sie wahrnehmen, was rundherum passiert. Und dass menschliche und medizinische Kriterien wieder wichtiger werden.»

\section{Die nächste Begegnung mit ...}

Am Ende jeden Monats stellt die Schweizerische Ärztezeitung eine Persönlichkeit vor, die sich im Gesundheitswesen engagiert. Im Januar schildert Daniel Lüthi seine Begegnung mit Rahel Kubik, Chefärztin für Radiologie am Kantonsspital Baden. 